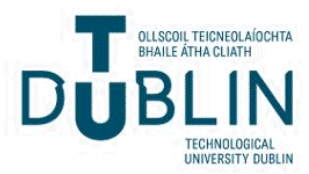

Technological University Dublin ARROW@TU Dublin

2008-01-01

\section{Anti-Isomorphisms and the Failure of Duality}

Brendan Goldsmith

Technological University Dublin, brendan.goldsmith@tudublin.ie

\author{
A. L. S. Corner \\ S. Wallutis \\ Universitat Duisberg-Essen
}

Follow this and additional works at: https://arrow.tudublin.ie/scschmatbk

\section{Recommended Citation}

Corner, A.L.S., Goldsmith, B. \& Wallutis, S.L. (2008). Anti-Isomorphisms and the Failure of Duality. Models, Modules and Abelian Groups , DeGruyter. https//doi.org/10.1515/9783110203035.315

This Book Chapter is brought to you for free and open access by the School of Mathematics at ARROW@TU Dublin. It has been accepted for inclusion in Book chapter/book by an authorized administrator of ARROW@TU Dublin. For more information, please contact arrow.admin@tudublin.ie, aisling.coyne@tudublin.ie, gerard.connolly@tudublin.ie. 


\title{
Anti-isomorphisms and the failure of duality
}

\author{
A. L. S. Corner, B. Goldsmith and S. L. Wallutis
}

\begin{abstract}
Groups and modules with isomorphic endomorphism rings are known, in certain cases, to be necessarily isomorphic. When such a ring isomorphism is replaced by an anti-isomorphism, the modules are often determined only up to isomorphism of certain duals. This type of situation is examined in a number of cases with special emphasis on the situation for mixed Abelian groups, where it is shown that no reasonable duality may exist.
\end{abstract}

Key words. Anti-isomorphism, Baer-Kaplansky theorem, mixed Abelian groups.

AMS classification. 20K30,20K21.

\section{Introduction}

A well-known theorem of Baer and Kaplansky ([1],[10]) states that Abelian $p$-groups are isomorphic if and only if their endomorphism rings are isomorphic. This theorem has been extended to other classes of Abelian groups and modules (see e.g. [9, 13]); in all cases the proofs are reasonably straightforward. The corresponding results for automorphism groups, i.e. that certain classes of modules are determined up to isomorphism by their automorphism groups, has also been the subject of a great deal of attention (see e.g. [11, 12, 2, 8]). It is a noticeable feature of these latter proofs that they are considerably more difficult than those relating to endomorphism algebras. Moreover the results contain an inbuilt 'duality' in that usually modules are determined not up to isomorphism but rather only up to isomorphism of the modules or their duals; the duals being a suitable group of homomorphisms. There is a 'halfway' case that has received some attention, viz the case of modules with anti-isomorphic endomorphism algebras. This situation is complex enough to admit the duality type outcome but is amenable, at least in some cases, to a more straightforward approach than is possible when dealing with automorphism groups. Note, of course, that if modules have anti-isomorphic endomorphism algebras, then composition of this anti-isomorphism with group inversion yields an isomorphism between the corresponding automorphism groups. Consequently, some of our results may be obtained from the corresponding results on automorphism groups; there are, however, situations, particularly involving the prime 2, where our approach yields results without amending the standard proof, whilst the corresponding result for automorphism groups is either quite complicated or unknown. We note that many of the results we display have been obtained previously (see e.g. [4, 14]) but our approach is quite different and more reminiscent of Kaplansky's approach in [10, Theorem 28].

There is an immediate problem in extending Kaplansky's Theorem from $p$-groups to mixed groups: the endomorphism rings of the quasi-cyclic group $\mathbb{Z}\left(p^{\infty}\right)$ and the

First author: Tony Corner died on September 3rd 2006, prior to the completion of this work. 
group of $p$-adic integers $\widehat{\mathbb{Z}}_{p}$ are isomorphic commutative rings. This led Kaplansky to comment [10, Exercise 96, p73]: This points up a critical difficulty in extending Theorem 28 to mixed modules. There are reasons for believing that a theory of duality is needed to clarify the situation. Perhaps it is true that when the rings of endomorphisms are isomorphic [or anti-isomorphic], the modules are isomorphic or "dual". In the final section of this paper we shall show that such a belief is rather naive: there exists a family of $2^{\aleph_{0}}$ pairwise non-isomorphic groups $\left\{G_{\alpha} \mid \alpha \in \mathbb{R}\right\}$ with $G_{\alpha} \leq G_{\beta}$ if $\alpha \leq \beta$, such that any pair have isomorphic and anti-isomorphic endomorphism rings. It is hard to see how a duality of the kind envisaged could be reconciled with such a situation.

Finally, we note that our notation and terminology is standard and may be found in $[6,7]$; an exception being that maps are written on the right.

\section{Torsion-free homogeneous separable groups}

There is, of course, a natural setting for anti-isomorphism in the context of Abelian group or module theory. Although our primary interest here is in Abelian groups, the module setting will be both natural and useful. Recall that if $G$ is a left $R$-module and $G^{*}$ denotes the $R$-module $\operatorname{Hom}_{R}(G, R)$, then every endomorphism $\alpha \in \operatorname{End}_{R} G$ induces a map $\alpha^{*} \in \operatorname{End}_{R} G^{*}$ by the rule

$$
f \alpha^{*}=\alpha \circ f \quad\left(f \in G^{*}\right)
$$

The mapping ( $)^{*}: \operatorname{End}_{R} G \rightarrow \operatorname{End}_{R} G^{*}$ is clearly an anti-homomorphism, so its composite with the corresponding ( $)^{*}: \operatorname{End}_{R} G^{*} \rightarrow \operatorname{End}_{R} G^{* *}$ is a homomorphism ()$^{* *}: \operatorname{End}_{R} G \rightarrow \operatorname{End}_{R} G^{* *}$. Moreover, there is a natural homomorphism $\iota: G \rightarrow G^{* *}$ given by the evaluation map

$$
f(g \iota)=g f \quad\left(g \in G, f \in G^{*}\right) ;
$$

recall that $G$ is said to be reflexive if this canonical map $\iota$ is an isomorphism. It is well known that these canonical homomorphisms constitute a natural transformation $i d \rightarrow()^{* *}$ in the sense that:

Lemma 2.1. For any $R$-module $G$ and $\alpha \in \operatorname{End}_{R} G$, the canonical homomorphism $\iota: G \rightarrow G^{* *}$ satisfies

$$
(g \iota) \alpha^{* *}=(g \alpha) \iota \quad(g \in G)
$$

Proof. Since $\alpha^{* *} \in \operatorname{End}_{R} G^{* *}$ and $g \iota \in G^{* *}$, we have $(g \iota) \alpha^{* *}=\alpha^{*} \circ(g \iota) \in G^{* *}$. Thus if $f \in G^{*}$, one has from (1) and (2) that

$$
f\left((g \iota) \alpha^{* *}\right)=\left(f \alpha^{*}\right)(g \iota)=g(\alpha \circ f)=(g \alpha) f=f((g \alpha) \iota) .
$$

Since $f$ was arbitrary we have the desired result.

It is now rather easy to establish the fundamental fact underlying all discussions of anti-isomorphisms in this context. 
Proposition 2.2. If $G$ is a reflexive $R$-module, then $\operatorname{End}_{R} G$ and $\operatorname{End}_{R} G^{*}$ are antiisomorphic.

Proof. From equation (3) in Lemma 2.1 above, we have that $(g \iota) \alpha^{* *}=(g \alpha) \iota \quad(g \in$ $G, \alpha \in \operatorname{End}_{R} G$ ), and now, since $G$ is assumed to be reflexive, we may identify $G$ with $G^{* *}$ so that $\iota$ becomes the identity. Then (3) simply asserts that $\alpha^{* *}=\alpha$, so that the composite $\operatorname{End}_{R} G \stackrel{()^{*}}{\longrightarrow} \operatorname{End}_{R} G^{*} \stackrel{()^{*}}{\longrightarrow} \operatorname{End}_{R} G$ is the identity. But $G$ being reflexive implies that $G^{*}$ is also reflexive and so, by symmetry, the two maps ()* are inverse anti-isomorphisms. In particular, $\operatorname{End}_{R} G$ and $\operatorname{End}_{R} G^{*}$ are anti-isomorphic.

Our principal objective in the remainder of this section is to establish a converse of the above Proposition when working with homogeneous separable groups; this restriction is quite natural since it is well known (see e.g. [3, IV Corollary 2.10]) that dual groups of the form $\operatorname{Hom}(G, \mathbb{Z})$ are always separable. It is also inevitable that in some situations one must invoke a further restriction requiring the type of the homogeneous group to be idempotent: if $S \leq \mathbb{Q}$ is a rational group which is not of idempotent type, then $S^{\aleph_{0}}$ is not separable homogeneous (see e.g. [7, Lemma 96.4]).

So suppose now that $G, H$ are homogeneous separable groups of type $R, S$ respectively with anti-isomorphic endomorphism rings. We need one final piece of notation: if $X$ is a homogeneous group of type $S$ then we set $X^{*}=\operatorname{Hom}(X, S)$ and $X_{*}=\operatorname{Hom}(S, X)$. The remainder of the section is devoted to showing:

Theorem 2.3. Let $G$ and $H$ be homogeneous separable groups of types $R, S$ respectively. If the endomorphism rings of $G$ and $H$ are anti-isomorphic then:

(i) the reduced types of $R, S$ coincide: $R_{0}=S_{0}$,

(ii) $G_{*} \cong H^{*}$ and $H_{*} \cong G^{*}$,

(iii) if the type $R$ of $G$ is idempotent then $G$ is $R$-reflexive.

Proof. Let $G, H$ be as above and let $\left(r_{1}, \ldots, r_{n}, \ldots\right)$ and $\left(s_{1}, \ldots, s_{n}, \ldots\right)$ be characteristics representing $R, S$ respectively. Then $r_{n}=\infty$ if and only if multiplication by $p_{n} 1_{G}$ is a unit in End $G$. However since anti-isomorphisms also preserve units, we immediately deduce that $s_{n}=\infty$ if and only if $r_{n}=\infty$, which is equivalent to saying that $R_{0}=S_{0}$.

Since $G$ is homogeneous separable of type $R$, we may choose a direct decomposition $G=G_{0} \oplus R g_{0}$, where $g_{0} \neq 0$. Let $\pi$ be the projection of $G$ onto $R g_{0}$ along $G_{0}$. Then, in the standard way, we may identify $\operatorname{Hom}\left(R g_{0}, G\right)$ with $\pi E$, where $E=$ End $G$. Similarly, $\operatorname{Hom}\left(G, R g_{0}\right)$ may be identified with $E \pi$. Since there is an anti-isomorphism ()$^{\prime}:$ End $G \rightarrow$ End $H$, we have $\pi^{\prime} \in E^{\prime}=$ End $H$, and as $\pi$ is an indecomposable idempotent in $E, \pi^{\prime}$ is an indecomposable idempotent in $E^{\prime}$. Therefore the summand $H \pi^{\prime}$ has the form $S h_{0}$ for some $h_{0} \neq 0$ and $H=H_{0} \oplus S h_{0}$, where $H_{0}=\operatorname{Ker} \pi^{\prime}$. Clearly, the anti-isomorphism maps $\pi E$ isomorphically onto $E^{\prime} \pi^{\prime}$ and $E \pi$ isomorphically onto $\pi^{\prime} E^{\prime}$. Thus $G_{*}=\operatorname{Hom}(R, G) \cong \pi E \cong E^{\prime} \pi^{\prime}=\operatorname{Hom}\left(H, S h_{0}\right) \cong H^{*}$. Similarly, $H_{*}=\operatorname{Hom}(S, H) \cong \pi^{\prime} E^{\prime} \cong E \pi \cong \operatorname{Hom}\left(G, R g_{0}\right) \cong G^{*}$. This establishes (ii). 
Finally, suppose that $R$ is an idempotent type so that $R=R_{0}$. Consider any $g \in$ $G, f \in G^{*}$ and let $g f=r \in R$. Denote by $g \rho, f \lambda$ the unique elements of $\pi E$ and $E \pi$ respectively, with

$$
g_{0}(g \rho)=g \text { and } x(f \lambda)=(x f) g_{0} \quad(x \in G, x f \in R) .
$$

Then $g_{0}(g \rho)(f \lambda)=g(f \lambda)=(g f) g_{0}=r g_{0}$ i.e.

$$
\text { if } g \in G, f \in G^{*} \text { and } g f=r \text {, then }(g \rho)(f \lambda)=r \pi \text {. }
$$

Now apply the anti-isomorphism ()$^{\prime}$ to the identity $(g \rho)(f \lambda)=r \pi$ of $(5)$ re-written in the form $n(g \rho)(f \lambda)=m \pi$, where $r=m / n$ and $m, n$ are integers. Then one gets $n(f \lambda)^{\prime}(g \rho)^{\prime}=m \pi^{\prime}$. However, since $R$ is of idempotent type, the integer $n$ consists only of prime factors $p_{n}$ corresponding to the places in the type of $R$ in which $\infty$ occurs. As the reduced types of $R, S$ are equal by (i), the occurrence of an $\infty$ in $R$ corresponds exactly to that in $S$ and so one may divide across this last equation to obtain $(f \lambda)^{\prime}(g \rho)^{\prime}=r \pi^{\prime}$.

Since $R$ is of idempotent type, $G_{*} \cong G$ so we have an isomorphism $G \stackrel{\rho}{\longrightarrow} \pi E$, which yields an isomorphism $\iota: G \rightarrow H^{*}$. Also $G^{*} \cong H_{*} \hookrightarrow H$, so we have a monomorphism $\kappa: G^{*} \hookrightarrow H$. But now it follows that $(f \kappa)(g \iota)=r$. Thus, if we identify $G^{*}$ in $H$ so that $\kappa$ becomes the identity, we retrieve equation (2) above; in other words, the isomorphism $\iota$ is none other than the canonical homomorphism $G \rightarrow G^{* *}$. Therefore $G$ is reflexive.

Remark 2.4. (i) It is not clear that one obtains reflexivity in the case where the type is not idempotent. Certainly the argument above fails at the key point where multiplication by the element $r \in R$ is preserved by the anti-isomorphism.

(ii) An examination of the proof of Theorem 2.3 shows that one does not require the full strength of the hypothesis that the groups be homogeneous and separable. In fact, the key property used is that, for each group, any rank-1 direct summand is of a fixed type (but not, of course, necessarily of the same type for the different groups). Thus, by a well-known theorem of Mishina (see e.g. [7, Proposition 96.2]), the result can be extended to include inter alia vector groups of the form $V=\prod R$, where $R$ is a fixed rank-1 group, even when $R$ is not of idempotent type; as noted above, in such circumstances $V$ is neither homogeneous nor separable.

It is rather easy to show that one cannot replace the groups $G_{*}$ and $H_{*}$ in the Theorem by $G$ and $H$ :

Example 2.5. Let $R$ be a rank-1 group with type $(1,1,1, \ldots)$ and let $G=R^{\omega}, H=$ $R^{(\omega)}$. Then there is an anti-isomorphism between End $G$ and End $H$ (via matrices) but $G^{*}=\operatorname{Hom}(G, R)=\bigoplus_{\omega} \operatorname{Hom}(R, R)=\mathbb{Z}^{(\omega)} \nsucceq H$ and $H^{*}=\operatorname{Hom}\left(\bigoplus_{\omega} R, R\right)$ $\cong \prod_{\omega} \operatorname{Hom}(R, R)=\mathbb{Z}^{\omega} \neq G$.

\section{3 -Groups and Mixed Groups}

The situation for separable $p$-groups is similar to, but more involved than, that for homogeneous separable torsion-free groups. Although the final outcome may be pre- 
sented so that it seems not to involve a duality - two separable $p$-groups with antiisomorphic endomorphism rings are necessarily isomorphic - there is, in fact, a strong duality at play here but the nature of $p$-groups allows one to actually deduce isomorphism from the duality. The result we present below is a special case of a complete characterization of the situation given by the first author in an unpublished manuscript dating back to the early 1960's. Since several other versions of the results for $p$-groups are now available (see in particular [4]), we present only an outline proof which is much influenced by, but somewhat simpler than, Liebert's approach to automorphism groups [12].

Theorem 3.1. If $G$ and $H$ are unbounded reduced separable p-groups with End $G$ anti-isomorphic to End $H$, then $G \cong H^{*}$ and $H \cong G^{*}$, where $(-)^{*}$ denotes the adjoint group $t\left(\operatorname{Hom}\left(-, \mathbb{Z}\left(p^{\infty}\right)\right)\right)$.

As indicated above, one can actually deduce from this that $G$ and $H$ are isomorphic; in fact $G, H$ must be torsion-complete $p$-groups with each Ulm invariant equal to 1 (see e.g. [5, Lemma 2.2]).

Proof. Since $G$ is an unbounded reduced separable $p$-group, for each $i \geq 1$ we may choose, as in [10, Theorem 28], direct decompositions $G=S_{1} \oplus \cdots \oplus S_{i} \oplus T_{i}$ where

(a) each $S_{i}$ is cyclic of order $p^{n(i)}$, with $n(i)$ increasing monotonically;

(b) $T_{1}>T_{2}>\ldots$;

(c) $S_{j}<T_{i}$ for $j>i$.

Choose generators $x_{i}$ for $S_{i}$ and idempotents $e_{i} \in$ End $G$ mapping $S_{i}$ identically to $S_{i}$ and annihilating the other summands. Since $G$ is separable we may define endomorphisms $e_{i j}$ such that $e_{i j}$ maps $x_{i}$ to $x_{j}$ if $i>j$ and maps $x_{i}$ to $p^{n(j)-n(i)} x_{j}$ if $i<j$; in either case the complementary summand is annihilated. Note that $e_{i} e_{i j}=e_{i j} e_{j}=$ $e_{i j}$. For convenience, denote End $G$ by $A$ and End $H$ by $B$ and let $\Phi$ be the antiisomorphism from $A$ onto $B$. Set $e_{i} \Phi=f_{i}$ so that $f_{i}$ is again an idempotent. Moreover, since $e_{i} A e_{i} \cong \operatorname{End}\left(G e_{i}\right)=\operatorname{End}\left(\left\langle x_{i}\right\rangle\right)$, it follows from $e_{i} A e_{i} \cong f_{i} B f_{i} \cong \operatorname{End}\left(H f_{i}\right)$, that $H f_{i}$ must also be cyclic of the same order $p^{n(i)}$. Let $H f_{i}=\left\langle y_{i}\right\rangle$.

Now consider the directed system of groups $\left\{e_{i} A, \pi_{i j}\right\}$ where for $i<j \pi_{i j}$ is given by $e_{i} \alpha \mapsto e_{j i} e_{i} \alpha$ for each $\alpha \in A$. Notice that since $e_{j i} e_{i}=e_{j} e_{j i}$, the expression $e_{j i} e_{i} \alpha$ is actually in $e_{j} A$. As we have observed previously, $e_{i} A$ is isomorphic to the group $\operatorname{Hom}\left(x_{i}, G\right)$ and hence the evaluation map $e_{i} \alpha \mapsto x_{i} e_{i} \alpha$ is a natural isomorphism of $e_{i} A$ onto $G\left[p^{n(i)}\right]$. The naturality of this map ensures that the directed systems $\left\{e_{i} A, \pi_{i j}\right\}$ and $\left\{G\left[p^{n(i)}\right], \iota_{i j}\right\}$, where $\iota_{i j}$ denotes inclusion, are isomorphic and hence the corresponding direct limits are isomorphic: $\lim _{\longrightarrow} e_{j} A \cong \lim _{\longrightarrow} G\left[p^{n(j)}\right] \cong G$.

Now define endomorphisms $f_{i j} \in B$ by setting $f_{i j}=e_{j i} \Phi$; note the change in order of the subscripts. By applying the anti-isomorphism $\Phi$ to the relations connecting the elements $e_{i j} \in A$, one obtains corresponding relations $f_{i} f_{i j}=f_{i j} f_{j}$. Consider the directed system $\left\{B f_{i}, q_{i j}\right\}$ where the maps $q_{i j}$ are given by $q_{i j}: \beta f_{i} \mapsto \beta f_{i} f_{i j}=$ $\beta f_{i j} f_{j}$. We claim that the directed systems $\left\{e_{i} A, \pi_{i j}\right\}$ and $\left\{B f_{i}, q_{i j}\right\}$ are isomorphic. This follows immediately from the commutivity, for each $n$, of the diagrams: 


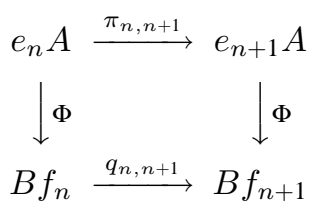

Thus, if we can establish that the limit of the directed system $\left\{B f_{i}, q_{i j}\right\}$ is isomorphic to $t\left(\operatorname{Hom}\left(H, \mathbb{Z}\left(p^{\infty}\right)\right)\right)$, where this latter is regarded as the direct limit of the socles $H^{*}\left[p^{n(i)}\right]$ with inclusions as the connecting maps, we are finished.

Take a presentation of the quasi-cyclic group $\mathbb{Z}\left(p^{\infty}\right)=\left\langle z_{1}, z_{2}, \ldots\right\rangle$ where $p^{n(1)} z_{1}=$ $0, p^{n(2)-n(1)} z_{2}=z_{1}, \ldots$ Now define, for each $n$, a map $\theta_{n}: H^{*} \rightarrow \mathbb{Z}\left(p^{\infty}\right)$ by $y_{n} \theta_{n}=$ $z_{n}$ with $\theta_{n}$ annihilating the complement of $\left\langle y_{n}\right\rangle$. Note that $\gamma_{k}: \beta f_{k} \mapsto \beta f_{k} \theta_{k}(\beta \in B)$ is a natural map taking $B f_{k}$ isomorphically onto $H^{*}\left[p^{n(k)}\right]$ since $B f_{k} \cong \operatorname{Hom}\left(H,\left\langle y_{k}\right\rangle\right)$. The existence of the isomorphism we are seeking to establish is equivalent to showing that, for each $k$, the diagrams below are commutative:

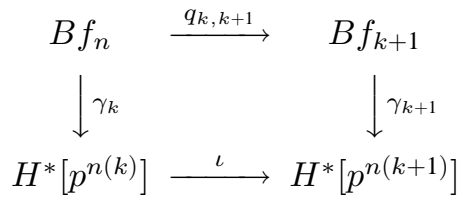

This follows from a simple diagram chase: if $h \in H$ and $\beta \in B$, then $h \beta$ can be expressed in the form $h \beta=r_{k} y_{k}+h^{\prime}$, so that $h \beta f_{k} q_{k, k+1}=r_{k} p^{n(k+1)-n(k)} y_{n+1}$. Applying $\gamma_{k+1}$ to this expression yields $r_{k} p^{n(k+1)-n(k)} z_{k+1}=r_{k} z_{k}$ and this is identical to the expression $h \beta f_{k} \gamma_{k}$. This completes the proof.

The situation for mixed groups with anti-isomorphic endomorphism rings is, however, vastly more complicated, even when the torsion part is a $p$-group. As noted in the introduction, Kaplansky felt that it was likely that the situation could be clarified by the use of a duality. Our next result shows that such a hope was essentially naive: the groups exhibited below have pairwise anti-isomorphic (and isomorphic) endomorphism rings, yet the containment relation between the groups is order-isomorphic to the real numbers.

Theorem 3.2. There exists a mixed group $G$ (which is even a p-adic module) together with a family of subgroups $G(\xi)$ indexed by a real parameter $\xi, 0<\xi \leq 1$ with the following properties:

(a) $G(\xi) \nRightarrow G\left(\xi^{\prime}\right)$ if $0<\xi<\xi^{\prime} \leq 1$;

(b) $\operatorname{End}(G(\xi)) \cong$ End $G$ for $0<\xi \leq 1$;

(c) End $G$ admits an anti-isomorphism.

Proof. Let $P=\prod_{i=1}^{\infty}\left\langle a_{i}\right\rangle$ be the direct product of finite cyclic groups $\left\langle a_{i}\right\rangle$ of order $p^{i}$. As usual we identify $a_{i}$ with the element $\left(0, \ldots, 0, a_{i}, 0, \ldots\right)$ of $P$, so that the elements $a_{i}$ generate a subgroup $S$ of $P$ which may be identified with the direct sum of the cyclic groups $\left\langle a_{i}\right\rangle$. Let $\widehat{S}$ denote the $p$-adic completion of $S$; clearly $\widehat{S} \leq P$. 
Define $G$ and $G(\xi)(0<\xi \leq 1)$ as subsets of $\widehat{S}$ in the following way: set $G=\widehat{S}$ and define $G(\xi)$ by $g=\left(g_{1}, g_{2}, \ldots\right) \in G(\xi)$ if and only if there exists an integer $k$ such that the height

$$
h\left(g_{k+i}\right) \geq \xi i \text { for } i=1,2, \ldots \text {. }
$$

It is easily verified that the $G(\xi)$ are subgroups of $\widehat{S}$, that $T=G(1)$ is the torsioncompletion of $S$, or equivalently the maximal torsion subgroup of $\widehat{S}$ and that we have the inclusion

$$
S \subset T \subset G\left(\xi^{\prime}\right) \subset G(\xi) \subset G \subset P \text { for } 0<\xi<\xi^{\prime}<1 .
$$

To simplify matters we write also $G(0)=G$.

First we show that

(A) The quotient group $G(\xi) / S$ is divisible for $0 \leq \xi \leq 1$.

It will be enough to prove that if $g \in G(\xi)$, then there exists an element $g^{\prime} \in G(\xi)$, with $g-p g^{\prime} \in S$. We shall prove this only in the case $0<\xi \leq 1$; the case $\xi=0$ is immediate since $G(0)=\widehat{S}$. Suppose then that $g$ satisfies condition (1) for some $k$. Let $n$ be the least integer not less than $\xi^{-1}$. Then, for $i=1,2, \ldots$ we have

$$
h\left(g_{k+n+i} \geq \xi(n+i) \geq 1+\xi i\right.
$$

consequently $\left\langle a_{k+n+i}\right\rangle$ contains an element $g_{k+n+i}^{\prime}$ with $p g_{k+n+i}^{\prime}=g_{k+n+i}$. It follows from (3) that $h\left(g_{k+n+i}^{\prime}\right) \geq \xi i$ for $i=1,2, \ldots$ and therefore $G(\xi)$ contains the element $g^{\prime}=\left(0, \ldots, g_{k+n+1}^{\prime}, g_{k+n+2}^{\prime}, \ldots\right)$. Clearly $g-p g^{\prime} \in S$.

Let $\phi$ be an arbitrary homomorphism $S \rightarrow P$. Then

$(B)$ for $0 \leq \xi \leq 1, \phi$ can be extended to a homomorphism $\bar{\phi}: G(\xi) \rightarrow P$ in precisely one way.

Since $P$ is reduced and, by (A), $G(\xi) / S$ is divisible, there can be at most one such extension $\bar{\phi}$. Moreover, since $G(\xi) \leq G$ for $0 \leq \xi \leq 1$, we need only prove that $\phi$ can be extended to a homomorphism $\bar{\phi}: G \rightarrow P$. This, however, follows immediately by continuity since $P$ is itself complete in the $p$-adic topology.

Now observe that

$(C)$ if $\phi$ is an arbitrary homomorphism $G(\xi) \rightarrow P$ for some $\xi, 0 \leq \xi \leq 1$, then $G(\xi) \phi \leq G(\xi)$.

Again we consider only the case $0<\xi \leq 1$. Let $g \in G(\xi)$. Then $g$ satisfies (1) for some integer $k$. It follows from (B) that for $j=1,2, \ldots$

$$
(g \phi)_{k+j}=\sum_{i=1}^{\infty}\left(g_{i} \phi\right)_{k+j}
$$

where all but a finite number of the terms of the sum are zero. We deduce that $h\left((g \phi)_{k+j}\right) \geq \min _{i} h\left(\left(g_{i} \omega\right)_{k+j}\right)$. For $1 \leq i \leq k$, we have $p^{i} g_{i}=0$ and, therefore, $p^{i}\left(g_{i} \phi\right)_{k+j}=0$, whence $h\left(\left(g_{i} \phi\right)_{k+j}\right) \geq k+j-i \geq j \geq \xi j$; for $k<i \leq k+j$, we have that $h\left(g_{i}\right) \geq x$, where $x$ is the least integer not less than $\xi(i-k)$; it follows that $p^{i-x} g_{i}=0$ and so $p^{i-x}\left(g_{i} \phi\right)_{k+j}=0$; consequently $h\left(\left(g_{i} \phi\right)_{k+j}\right) \geq k+j-(i-x)=$ $k+j-i+x \geq(k+j-i)+\xi(i-k) \geq \xi(k+j-i)+\xi(i-k)=\xi j$. For $k+j<i$, we have $h\left(\left(g_{i} \phi_{k+j}\right) \geq h\left(g_{i}\right) \geq \xi(i-k)>\xi j\right.$. Hence $h\left((g \phi)_{k+j}\right) \geq \min _{i} h\left(\left(g_{i} \phi\right)_{k+j}\right) \geq \xi j$; therefore $g \phi \in G(\xi)$.

We are now ready to prove (a), (b) and (c). 
(a) Suppose, if possible that $\phi: G\left(\xi^{\prime}\right) \rightarrow G(\xi)$ is an isomorphism where $0<\xi<$ $\xi^{\prime} \leq 1$. If we regard $\phi$ as a homomorphism of $G\left(\xi^{\prime}\right)$ into $P$, then it follows from $(C)$ that $G(\xi)=G\left(\xi^{\prime}\right) \phi \leq G\left(\xi^{\prime}\right)$. Now $G(\xi)$ contains the element $g=\left(g_{1}, g_{2}, \ldots\right)$ where for $i=1,2, \ldots, g_{i}=p^{[\xi i+1]} a_{i}$; here, as usual, $[x]$ denotes the integer part of the real number $x$. It follows from the inclusion $G(\xi) \leq G\left(\xi^{\prime}\right)$ that $g \in G\left(\xi^{\prime}\right)$, i.e. for some integer $k$ we have

$$
[\xi(k+i)+1]=h\left(g_{k+i}\right) \geq \xi^{\prime} i \quad \text { for } i=1,2, \ldots
$$

Consequently, $\xi k+1 \geq\left(\xi^{\prime}-\xi\right) i$ for $i=1,2, \ldots$; since, by hypothesis $\xi^{\prime}-\xi>0$, this is impossible. Thus we have shown that $G(\xi) \nRightarrow G\left(\xi^{\prime}\right)$.

(b) Let $0<\xi \leq 1$ and suppose that $\phi \in \operatorname{End}(G(\xi))$. Denote the restriction of $\phi$ to $T$ by $\phi^{\prime}$ so that $\varepsilon: \phi \mapsto \phi^{\prime}$ is a ring homomorphism $\operatorname{End}(G(\xi)) \rightarrow \operatorname{End} T$. By $(A)$ the kernel of $\varepsilon$ is 0 : any element of $\operatorname{Ker} \varepsilon$ induces a map from the divisible group $G((\xi)) / T$ into the reduced group $G((\xi))$ and hence is identically zero. Moreover, it follows from $(B)$ and $(C)$ that any element of End $T$ lifts to an endomorphism of $G((\xi))$, so that $\varepsilon$ is onto. Thus $\operatorname{End}(G(\xi)) \cong$ End $T$ as required.

(c) Since End $G \cong \operatorname{End}(G(1))$, it suffices to show that $\operatorname{End}(G(1))=$ End $T$ possesses an anti-automorphism. If $\phi \in$ End $T$, then $\phi$ is uniquely determined by its restriction to $S$ and thus we may represent $\phi$ as an $\omega \times \omega$ matrix obtained in the usual way from the equations $a_{i} \phi=\sum_{j=1}^{\infty} r_{i j} a_{j}$, where the right hand side is being interpreted as an element of $P$. Now let $\phi^{*}$ be the mapping $S \rightarrow P$ defined by $a_{i} \phi^{*}=\sum_{j=1}^{\infty} r_{j i} a_{j}$. Clearly $\phi^{*}$ is a well-defined homomorphism and corresponds to the classical transpose matrix of $\phi$. Moreover, from $(B), \phi^{*}$ may be extended to a map which we continue to denote by $\phi^{*}: T=G(1) \rightarrow P$ and, as $T$ is fully invariant, $\phi^{*}$ actually maps $T \rightarrow T$, i.e. $\phi^{*} \in$ End $T$. Clearly the assignment $\phi \mapsto \phi^{*}$ is an anti-homomorphism of End $T$ and since $\left(\phi^{*}\right)^{*}=\phi$, it is an anti-automorphism.

\section{References}

[1] R. Baer, Automorphism rings of primary abelian operator groups, Ann. Math. 44 (1943), 192277.

[2] A.L.S. Corner and B. Goldsmith, Isomorphic Automorphism Groups of p-adic Modules, in Abelian Groups, Module Theory and Topology, (ed. D. Dikranjian and L. Salce), Lecture Notes in Pure and Applied Mathematics, 201, 125-130, Marcel Dekker, New York (1998).

[3] P. Eklof and A. Mekler, Almost Free Modules, Set-theoretic Methods, North-Holland, 2002.

[4] G. D'Este, Abelian Groups with Anti-Isomorphic Endomorphism Rings, Rend. Sem. Mat. Univ. Padova 60 (1978), 55-75.

[5] K. Faltings, On the Automorphism Group of a Reduced Primary Abelian Group, Trans. Amer. Math. Soc. 165 (1972), 1-25.

[6] L. Fuchs, Infinite Abelian groups I, Academic Press, New York (1970).

[7] L. Fuchs, Infinite Abelian groups II, Academic Press, New York (1973). 
[8] B. Goldsmith and P. Zanardo, Endomorphism Rings and Automorphism Groups of Separable Torsion-Free Modules over Valuation Domains, in Abelian Groups, Module Theory and Topology, (ed. D. Dikranjian and L. Salce), Lecture Notes in Pure and Applied Mathematics, 201, 249-260, Marcel Dekker, New York (1998).

[9] G.J. Hauptfleisch, Torsion-free abelian groups with isomorphic endomorphism rings, Arch. Math. 24 (1973), 269-273.

[10] I. Kaplansky, Infinite Abelian Groups, University of Michigan Press, Ann Arbor, 1954 and 1969.

[11] H. Leptin, Abelsche p-Gruppen und ihre Automorphismengruppen, Math. Z. 73 (1960), 235253.

[12] W. Liebert, Isomorphic Automorphism Groups of Primary Abelian Groups, in Abelian Group Theory, Proceedings of the 1985 Oberwolfach Conference, 9-31, Gordon and Breach, New York (1987).

[13] W. May, Isomorphism of Endomorphism Algebras over Complete Discrete Valuation Rings, Math. Z. 204 (1990), 485-499.

[14] K.J. Wolfson, Anti-Isomorphisms of Endomorphism Rings of Locally Free Modules, Math.Z. 202 (1989), 151-159.

\section{Author information}

A. L. S. Corner, formerly of Worcester College, Oxford., England.

Email:

B. Goldsmith, School of Mathematical Sciences, Dublin Institute of Technology, Aungier Street, Dublin.2, Ireland.

Email: brendan.goldsmith@dit.ie

S. L. Wallutis, Fachbereich 6, Mathematik, Universität Duisburg-Essen, 45117 Essen, Germany.

Email: simone@wallutis.de 